\title{
SEMANTIC SEARCH OVER WIKIPEDIA DOCUMENTS MEANING OF QUERIES BASED PRE-TRAINED LANGUAGE MODEL
}

\author{
THARUN P
}

St Joseph's College of Engineering, India

\begin{abstract}
The previously trained on massive text corpora such as GPT-3 is powerful and has an open domain with more than 175 billion parameters. However, as our semantic search will make it possible to search with the keyword that will give you what it was searched for, it is still challenging for such models to train and get the accuracy level challenging model Coherently for prolonged passages of textual content, in particular at the same time as the models This focuses on the target area of the small figure. In the next few steps, the key formulas for domain precise content materials will become more and more complex. Wikipedia's semantic file search, to calculate the semantic relevance of language text, requires multiples of data set search. Which is, Important common sense and global knowledge on specific topics. By recommending Semantic Search Analysis (SSA), which is a fully specialized technique for representing text in the main superior domain obtained from Wikipedia. Using the previously trained strategy, we mainly construct the average value of the content of the text explicitly on the adaptive model from Wikipedia. Results display that our version outperforms different models.
\end{abstract}

KEYWORDS: GPT, Deep learning, Semantic Search, RNN, Learning Predictions

Received: May 25, 2021; Accepted: Jun 15, 2021; Published: Jul 02, 2021; Paper Id.: IJCSEITRDEC20212

\section{INTRODUCTION}

A better understanding of consumer goals is critical to improving the best search engines and optimizing the consumer experience. Traditional search engines mainly rely on matching key phrases in the file with search queries. From an exclusive statement. Users also need to remember multiple ways of arranging question clusters so that they can get the right statistics they need. As a result, semantic search engines have emerged to better serve our customers.

Recently, short-term long-term memory (LSTM) networks have shown excellent overall performance in various types of natural language processing tasks, including annotated images, translation programs, and semantic search. That is to identify applicable issues from existing issues and find solutions for new requests in the community response forum. Inspired by this challenge, that proposes a reconstructed neural network model based on which predicts the evaluation version of the semantic relationship based entirely on the similarity between the related parent sentence and the semantic research. We have also confirmed that the version acceptance in the various set and generated transformers are running tests to verify the directionality of various models.

This article contains the following content:

- By learning the unique technology of semantic search analysis method. In this field, the consumer question contains far fewer records than the final result search. Therefore, this article recommends pruning the final results of the search report, and in many cases consolidating consumer issues and related documents to stabilize the record number. 
- We will input a search query and the model will return relevant movie titles with "Relevance \%" which is the similarity score. The higher the similarity score, the more similar the query to the document at the given index.

- Conduct experiments to test the application of our version and evaluate our version on another version with a special version of the GPT architecture.

The traditional language model cannot fully capture semantic statistics without considering the components of the collection. Recurrent neural network (RNN), which isn't always like a regular neural network, introduces consistent circulate its model simply so it can gadget collection statistics. RNN indicates great basic overall performance in processing many tasks involve collecting data, but RNNs have long-term dependency issues and lengthy technical paragraphs that contain too much statistical information. The LSTM architecture is also proposed based on these activities that data solves the problem of long-term dependence by introducing a memory that can store statistical data for a long time in the RNN structure. The pretrained trained is currently used to infer complexity statistics to extract sentence-level and sentence-level semantic vectors from contextual queries.

Latent semantic analysis is another proprietary statistical technique that uses sentence match statistics from a large corpus of unlabeled corpus. Which no longer uses human-created knowledge. Instead, use Singular Value Analysis to "learn" the digit in a sentence through a file matching with the matrix. That finds "Hidden concept" of a sentence. The terms and meanings of the files are then displayed in the areas explained by those concepts.

This is some other in basic terms statistical approach, which leverages phrase co-incidence statistics from a huge unlabeled corpus of text. GPT does now no longer use any express human-prepared knowledge; rather, it "learns" its illustration with the aid of using making use of Singular Value Use file matching matrix to decompose into phrases. GPT is essentially a dimensional discount method, which identifies some of the significant largest dimensions in the data that should conform to the "hidden concept". The meaning of phrases and documents are presented within the scope of the description using these concepts.

Databases including WordNet or Rogers Thesaurus encode key family members between phrases, including synonyms, or which is word related to the meaning of the word and word segmentation of API. A method mainly based on such sources assigns a phrase with textual content to the meaning of the phrase and uses it as a concept. The source provides almost no record of the specific meaning of the phrase, so it is almost impossible to make the phrase too clear. A small part of the language vocabulary is more effective. In particular, such sources contain multiple correct names, new words, jargon and terminology that are unique in the field. In addition, these sources have a strong lexical orientation, because they mainly contain the register of symbolic expression, but the global knowledge is insufficient. Generally speaking. The strategy of symbolic sentences requires additional classes to process longer texts, for example, the similarity calculation of several volumes of text in order to evaluate each sentence 1 with text content and each sentence with opposite text content.

\section{EXPLICIT SEMANTIC ANALYSIS}

\section{A. Neural Networks}

For example, if I search for articles about the stock market and search for "rising stocks", but I have an article that says "rising stocks", searching verbatim becomes difficult. Link to the corresponding article here. Obviously, even if the information is there, my results are useless. This makes a huge dataset to collect and retrieve according to the data and match them related to 
the words.

The improvement of a semantic seek engine makes use of GPT based (pre-skilled transformer) embeddings which has advanced the hunt engine and the use of GPT embeddings. Both of the works used cosine similarity to compute the similarity rating of question and documents. My paintings makes use of GPT embeddings however makes use of neural community to discover the similarity rating.

Knowledge Graphs can offer wealthy structural data approximately entities. It additionally captures kinds of relationships with neighboring entities and the general symbolic context. Knowledge embeddings discovered over a understanding graph helps capture the understanding semantics for entities. We can complex in element on how understanding context round conceptual entities and ambiguous entities may be used.

- Conceptual entities: Semantic illustration of entities that aren't a part of pre-skilled transformer vocabularies is approximated with the aid of using blending the embedding discovered over their sub-phrases tokens. This approximation is a bottleneck to analyze the particular semantic that means for such entities, (i.e.) "Greenhouse effect", "Bread is so fluffy", and "Refraction", etc. Knowledge context for such conceptual entities may be described as wealthy informative context gift withinside the information graph. Infusing such wealthy informative context for such entities have to have a wonderful ability to enhance overall performance for downstream applications. Further, the dataset for the domain-particular assignment could be ruled with the aid of using domain-particular entities, and therefore there could be greater ability to enhance overall performance with the aid of using infusing wealthy informative context for such entities from the domain-particular information graph.

- Ambiguous entities: Entities like often used verbs, adjectives, and nouns can be afflicted by phrase polysemy or homophony. Where an entity may have exclusive meanings or exclusive entities may have the identical that means primarily based totally at the context. For example, the verb "bread" may have exclusive meanings, and exclusive entities "fluffy" and "eat" can precisely have the identical that means relying on the context. Knowledge context for such bold entities may be derived from the use of steps. First, predicting the disambiguated feel of ambiguous entities, and then, leveraging the information graph to derive the information context of the disambiguated feel, which could encompass its relationships with comparable senses and different lexical entities. Infusing information-context of disambiguated senses for such ambiguous entities have to have vast ability to enhance the model's overall performance.

\section{B. Pretrained transformer}

Our technique is stimulated with the aid of using the choice to enhance textual content illustration with large quantities of global wide know-how. The text is formed as a weighted combination of a set of predefined herbal standards, written with human use and easy to interpret. To do this, we will use the standards described in Wikipedia articles. (e.g.) LEMON ON THE ORANGE CAR. A crucial benefit of our technique is for that reason the usage of significant quantities of incredibly prepared human know-how that encodes on Wikipedia. Wikipedia is constantly improving, so its amplitude and intensity that will increase regularly over time.

The answer to this question is semantic search. With the latest findings of NLP research, language models can be trained on a large number of documents. The model can then represent the document in terms of its "Bread" content. This 
includes the ability to search for documents with semantically similar content. The million phrases in $(60000+$ plus-articles $)$ By improvement method provides top-notch quality, and current research on Wikipedia is comparable to other related semantic accuracy.

\section{Study and Analysis of Knowledge}

The concept that used in these strategies to construct a semantic interpreter which creates a semantic interpreter that directly compares snippets of the herbal language text content with a series of weighted Wikipedia ideas, sorted by the relevance of the introduction. Therefore, the input text is expressed as a vector of GPT explaining ideas, which is called an explanation vector. The which means of a textual content fragment is for that reason This explained model of the phrases on how close it is to various ideas on Wikipedia. By calculating the semantic relationship of the text, and then using ideas (for example, using the trained measurement) to evaluate the size of its vector in the described area, the semantic evaluation expresses the thought based on human cognition on the side where we control the perception of reception, As a substitute for the "hidden thought" of implicit through semantic analysis.

\section{Training and approach}

To illustrate my approach, we show the 10 Wikipedia principles with the highest scores among the vectors used to interpret text content template fragments. Looked after withinside the reducing order in their score, the pinnacle ten principles are the maximum applicable ones for the enter textual content. This additionally suggests the maximum applicable Wikipedia principles for character phrases ("equipment" and "investor", respectively), at the same time as it makes use of the longer paragraph serves as an example of Matching translation vectors for fragments containing ambiguous phrases towards the matched words which are particularly interesting. This suggests the primary entries withinside the vectors for terms that incorporate ambiguous phrases "Fast" and" Furious". As may be without difficulty seen, our semantic interpretation method is able to appear phrase feel disambiguation, through thinking about ambiguous phrases withinside the context in their neighbors.

\section{RELATED WORK}

Quantifying the limitations of text semantic relations is the basis of many important tasks in computational linguistics, such as dis-ambiguating sentence meaning, searching for relevant word stats, grouping sentences and text content, and correcting errors. Fragments are the burden of phrases in the vector space the use of many resources and the use of semantic analysis converters (GPT), the first method is the simplest, but not the best reproduction, and the text is compared with several sentences, the text uses synonyms to convey similar messages. This method also has nothing to do with evaluating male or female phrases. These latter strategies try to circumvent this restriction.

The concept which contains members of the WordNet or Roget synonym code family between phrases, including synonyms and hypernyms. Some indicators have been described for calculating the ratio of multiple household used to the basic graphical form of these assets. The point is that creating lexical assets not only requires a lot of time and effort, but also requires knowledge of lexicography, so these assets contain a small part of language vocabulary. Such assets especially include several correct names, new words, jargon and specific fields. In addition, these resources have a strong vocabulary focus, mainly including recordings of personal phrases, but overall, there is little international experience. Strategies based primarily on WordNet are very similar to ESA, as each strategy drives the development of many ideas. On the one hand, 
methods mainly based on WordNet are naturally limited to suggestions made by individuals, and their models for evaluating longer texts require additional complexity. On the contrary, our technology treats every sentence and text almost equally. Second, considering phrases in our technical context can eliminate phrase ambiguity. The use of WordNet is not clear, because the sunset fact is limited to three sentences (brightness); in every GPT and Wikipedia idea is associated with a large amount of text. Finally, this is also known as Semantic Search. According to Wikipedia: Semantic search denotes search with meaning, as distinguished from lexical search where the search engine looks for literal matches of the query words or variants of them, without understanding the overall meaning of the query. (or text) as a weighted set of thoughts, and display the phrase in the WordNet collection for easy searching without weight. In addition, in WordNet, the meaning of each sentence is unique. Technically, the idea reflects unusual input components, creating a weighted, multifaceted representation of the text.

In contrast, from a layman's point of view, GPT is a statistical technique that uses phrase matching records from a large corpus of unlabeled text content. GPT no longer relies on artificially generated knowledge; instead which uses similar to the component used in sentences to "learn" the illustrations by using a file matching matrix to the keyword. GPT is essentially a dimensional discount method, which defines the maximum data size of some allocations. This must correspond to "hidden thoughts". Then compare the meanings of phrases and documents in the described area based on these ideas. The underlying semantic tendency is difficult to explain because, with the help of humans, calculated ideas cannot be easily converted into manipulated herbal ideas. The explicit semantic analysis technique we propose avoids this problem by presenting the meaning of the text content and separating the use of herbal thoughts with the help of humans.

Our method of evaluating the semantic relationship of sentences gives a fair evaluation of the similarity of the distribution. In fact, we used popular style evaluation methods in various herbal language documents to evaluate the meaning of idioms. However, the compilation of these documents is not random, but corresponds to the articles in the encyclopedia, each article revolves around a theme. In this article, we consider "semantic relationship" rather than "semantic similarity" or "semantic distance", which may be because he believes in his extensive research on kinship measurement that kinship beliefs are superior to similarity beliefs. They also believe that the computational linguistics package requires regular kinship measurements. For example, to clarify the meaning of a phrase, you can use any related phrase in the context, and now it is no longer just a comparable phrase. In addition, he believes that belief in semantic distance may be complicated by various methods used in the literature.

One way to solve this problem is to use clause insertion. Sentence embedding is a general term for many natural language processing (NLP) technologies that map sentences to real-number vectors. (Source: Wikipedia) In this article, we will mainly discuss how to get inline suggestions and how to compare some of them. These methods have been studied in recent years. Some existing methods for creating inline sentences are: Infer Sent General Sentence Encoder Medium glove tabs Medium BERT tabs Option BERT: Sentence tabs with Siamese BERT network Here we see the above list The implementation of the last two methods, the "medium BERT tab" and the -BERT (SBERT) clause, we will try to understand how these methods work and can be used.

\section{SYSTEM ARCHITECTURE, DESIGN AND IMPLEMENTATION}

\section{A. System Overview and Design}

Our method to estimating semantic relatedness of phrases is extremely paying homage to distributional similarity. Indeed, we evaluate the meanings of phrases via way of means of evaluating the prevalence styles throughout a huge series of herbal 
language files. However, the compilation of these documents is not arbitrary; on the contrary, these documents correspond to articles in the encyclopedia, and each article points to an unrelated topic. In this article, we focus on "semantic relations" rather than "semantic similarities." Or "semantic distance", which can also be used frequently in the literature. In his extensive research on kinship measurement, he believes that kinship beliefs are superior to similarity beliefs, because the former contains many unique and unique relationship forms, such as separation, antonymy, and deliberate association. Similarly, they believe that computational linguistics packages usually require an affinity measure, rather than a more rigorously described affinity measure. For example, related phrases in context can be used to make the meaning of the phrase too clear, and now they are no longer just comparable phrases. Additionally, argued that the belief of semantic distance is probably complicated because of the distinctive approaches it's been used withinside the literature.

This method expresses beyond the meaning of the text as a weighted vector of knowledge terms. It is important to note that the entries in this vector correspond to clear, human-defined concepts, rather than simple words that are usually ambiguous. Large amounts of hand-coded human knowledge, rather than defining concepts through statistical analysis of academic terminology corpus. Compared with CYC, our method simplifies the semantic interpretation process independent of manual coding inference rules. Most previous semantic interpretation methods clearly express the semantics of individual words and require additional complexity to represent longer texts. Instead, our method consistently displays the meaning of text, regardless of their length.

Table 4.1: The Effect of Feature Generation for Long Document

\begin{tabular}{|c|c|c|c|c|c|}
\hline \multirow{2}{*}{ DATASET } & \multicolumn{2}{|c|}{ Baseline } & \multirow{2}{*}{\multicolumn{2}{|c|}{ Wikipedia }} & \multirow{2}{*}{$\begin{array}{l}\text { Improv } \\
\text { e-ment }\end{array}$} \\
\hline & Micro & Macro & & & \\
\hline $\begin{array}{l}\text { Reuters- } 21578 \\
\text { (90 breads) }\end{array}$ & 0.925 & 0.874 & 0.932 & $+0.8 \%$ & $+1.5 \%$ \\
\hline $\begin{array}{l}\text { RCV1 } \\
\text { Industry-16 }\end{array}$ & 0.877 & 0.602 & 0.883 & $+0.7 \%$ & $+0.2 \%$ \\
\hline $\begin{array}{l}\text { RCV2 } \\
\text { Industry-10A }\end{array}$ & 0.642 & 0.595 & 0.645 & $+0.5 \%$ & $+3.7 \%$ \\
\hline $\begin{array}{l}\text { RCV3 } \\
\text { Industry-10B }\end{array}$ & 0.421 & 0.335 & 0.448 & $+6.4 \%$ & $+30.4 \%$ \\
\hline $\begin{array}{l}\text { RCV4 } \\
\text { Industry-10C }\end{array}$ & 0.489 & 0.528 & 0.523 & $+7.0 \%$ & $+7.2 \%$ \\
\hline $\begin{array}{l}\text { RCV5 } \\
\text { Industry-10D }\end{array}$ & 0.443 & 0.414 & 0.468 & $+5.6 \%$ & $+4.1 \%$ \\
\hline $\mathrm{RC}$ & 0.587 & 0.466 & 0.595 & $+1.4 \%$ & $-1.5 \%$ \\
\hline Indu & 0.648 & 0.605 & 0.641 & $-1.1 \%$ & $+1.2 \%$ \\
\hline $20 \mathrm{NG}$ & 0.854 & & 0.862 & & +6.1 \\
\hline
\end{tabular}

This eliminates enormous connections between articles and words by resetting the weight of concepts that are too small for a particular term to zero. 


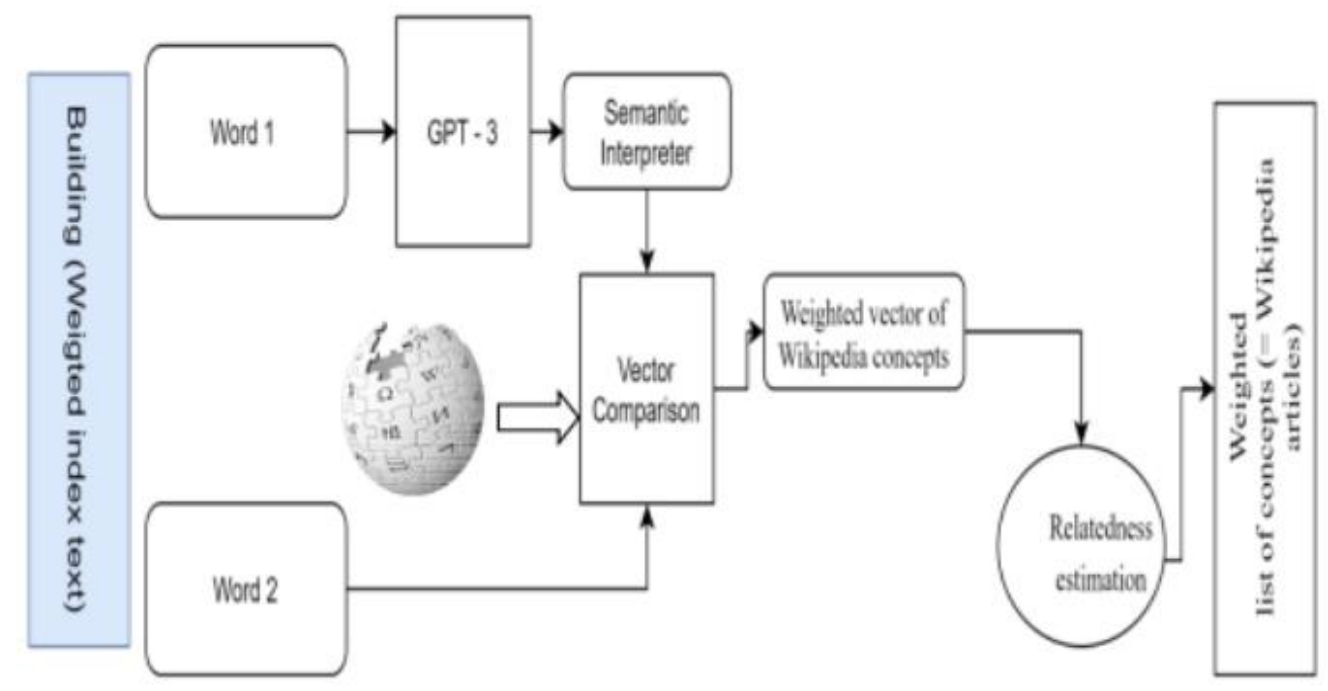

Figure 5.1: Knowledge-Based Semantic Interpreter

Figure 5.1 This depicts the standard method of classifying text. This describes the recommended structure for the generated function; note that the Create Function field replaces the function selection field in bold.

\section{B. Implementation Details}

Modern technology should enhance the pattern performance of massive LMs fine-tuned to goal domains. The instinct is that via way of means of focusing on the subsets of informative words, the early tiers can greater efficaciously seize the domain-particular traits after which steer the following refinement tiers. This suggests the effects in which we record the that we will see our method could make greater green use of the schooling facts in getting to know to generate excessive nice samples. For example, with the handiest $1 \mathrm{~K}$ schooling examples, our technique achieves similar effects with massive LMs educated on $30 \mathrm{~K}$ examples.

LSA (Latent Semantic Analysis), also known as LSI (Latent Semantic Index) LSA is a natural language processing technology that analyzes the relationship between a set of documents and the terms it contains by creating a set of concepts and assigns values to documents and terms. Based on the principle that words used in the same context often have similar meanings. A key feature of LSI is its ability to extract conceptual content from the text body by establishing associations between terms that appear in similar contexts.

Recent empirical upgrades because of switch gaining knowledge of language pre-trained model fashions have validated that rich, unsupervised pre-schooling is a crucial part of many language information systems. In particular, those consequences permit even low-useful resource obligations to advantage from deep unidirectional architectures. The fundamental contribution is similarly generalizing those findings to deep bidirectional architectures, permitting the equal pre-skilled version to efficiently address a vast set of NLP obligations.

\section{CONCLUSIONS}

Explicit Semantic Analysis—a semantic interpretation method for transformer language processing. In order to render computer systems with understanding approximately the world, we use Wikipedia to construct a semantic interpreter, which represents the means of texts in a completely high-dimensional area of understanding-primarily based ideas. These ideas correspond to Wikipedia articles, and our method presents a completely computerized manner to faucet into the collective 
understanding of tens and loads of lots of people. The idea primarily based illustration of textual content carries facts that cannot be deduced from the enter textual content alone and therefore supersede the traditional bag of phrases illustration. in particular crafted inference policies or counting on extra common-feel understanding bases. This turned into made viable via way of means of making use of trendy textual content class strategies to healthy report texts with applicable Wikipedia articles. to carry out function technology for textual content categorization yielded constant enhancements throughout a numerous variety of datasets. Recently, the overall performance of the quality textual content categorization structures has become similar, and former paintings usually performed small enhancements. Using Wikipedia as a supply of outside understanding allowed us to enhance the overall performance of textual content categorization throughout a numerous series of datasets.

\section{REFERENCES}

1. Antoine Bordes, Nicolas Usunier, Alberto Garc'iaDuran, Jason Weston, and Oksana Yakhnenko. 2013.

2. A. Abid, M. Farooqi, and J. Zou. Persistent anti-muslim bias in large language models. CoRR, abs/2101.05783, 2021.

3. S. L. Blodgett, S. Barocas, H. D. III, and H. M. Wallach. Language (technology) is power: A critical survey of "bias" in NLP. CoRR, abs/2005.14050, 2020.

4. T. Bolukbasi, K. Chang, J. Y. Zou, V. Saligrama, and A. Kalai. Man is to computer programmer as woman is to homemaker? debiasing word embeddings. CoRR, abs/1607.06520, 2016.

5. G. Branwen. GPT-3 Creative Fiction, 2021.

6. T. B. Brown, B. Mann, N. Ryder, M. Subbiah, J. Kaplan, P. Dhariwal, A. Neelakantan, P. Shyam, G. Sastry, A. Askell, S. Agarwal, A. Herbert-Voss, G. Krueger, T. Henighan, R. Child, A. Ramesh, D. M. Ziegler, J. Wu, C. Winter, C. Hesse, M. Chen, E. Sigler, M. Litwin, S. Gray, B. Chess, J. Clark, C. Berner, S. McCandlish, A. Radford, I. Sutskever, and D. Amodei. Language models are few-shot learners. CoRR, abs/2005.14165, 2020.

7. Sanjeev Banerjee and Ted Pedersen. Extended gloss overlaps as a measure of semantic relatedness. In IJCAI, pages 805-810, 2003.

8. Antoine Bosselut, Asli Celikyilmaz, Xiaodong He, Jianfeng Gao, Po-Sen Huang, and Yejin Choi. 2018. Discourse-aware neural rewards for coherent text generation. In NAACL, pages 173-184

9. Krzysztof Choromanski, Valerii Likhosherstov, David Dohan, Xingyou Song, Andreea Gane, Tamas Sarlos, Peter Hawkins, Jared Davis, Afroz Mohiuddin, Lukasz Kaiser, et al. 2021.

10.D. M. Jr., V. Prabhakaran, J. Kuhlberg, A. Smart, and W. S. Isaac. Extending the machine learning abstraction boundary: A complex systems approach to incorporate societal context. CoRR, abs/2006.09663, 2020.

11.Z. Kenton, T. Everitt, L. Weidinger, I. Gabriel, V. Mikulik, and G. Irving. Alignment of language agents. CoRR, abs/2103.14659, 2021.

12.A. Liu, M. Sap, X. Lu, S. Swayamdipta, C. Bhagavatula, N. A. Smith, and Y. Choi. On-the-fly controlled text generation with experts and anti-experts, 2021.

13. A. Holtzman, J. Buys, M. Forbes, and Y. Choi. The curious case of neural text degeneration. CoRR, abs/1904.09751, 2019.

14.A. Liu, M. Sap, X. Lu, S. Swayamdipta, C. Bhagavatula, N. A. Smith, and Y. Choi. On-the-fly controlled text generation with experts and anti-experts, 2021. 
15. P. P. Liang, I. M. Li, E. Zheng, Y. C. Lim, R. Salakhutdinov, and L. Morency. Towards debiasing sentence representations. CoRR, abs/2007.08100, 2020.

16. M. Sap, D. Card, S. Gabriel, Y. Choi, and N. A. Smith. The risk of racial bias in hate speech detection. In Proceedings of the 57th Annual Meeting of the Association for Computational Linguistics, pages 1668-1678, Florence, Italy, July 2019.

17. United Nations Office of the High Commissioner. Human Rights enhancing equality and countering discrimination, 2021.

18. B. Vidgen, A. Harris, D. Nguyen, R. Tromble, S. Hale, and H. Margetts. Challenges and frontiers in abusive content detection. In Proceedings of the Third Workshop on Abusive Language Online, pages 80-93, Florence, Italy, Aug. 2019.

19. A. Xu, E. Pathak, E. Wallace, S. Gururangan, M. Sap, and D. Klein. Detoxifying language models risks marginalizing minority voices. CoRR, abs/2104.06390, 2021.

20.Zhan Shi, Xinchi Chen, Xipeng Qiu, and Xuanjing Huang. 2018. Toward diverse text generation with inverse reinforcement learning. IJCAI

21. Mitchell Stern, William Chan, Jamie Kiros, and Jakob Uszkoreit. 2019. Insertion transformer: Flexible sequence generation via insertion operations.

22. Roman Novak, Michael Auli, and David Grangier.2016. Iterative refinement for machine translation.

23. Ratish Puduppully, Li Dong, and Mirella Lapata. 2019. Data-to-text generation with content selection and planning. In AAAI, volume 33, pages 6908-6915. 
